\title{
Major human milk oligosaccharides are absorbed into the systemic circulation after oral administration in rats
}

\author{
E. Vazquez ${ }^{1 *}$, A. Santos-Fandila ${ }^{1}$, R. Buck ${ }^{2}$, R. Rueda ${ }^{1}$ and M. Ramirez ${ }^{1}$ \\ ${ }^{1}$ Discovery RED, Abbott Nutrition, 18004 Granada, Spain \\ ${ }^{2}$ Discovery RED, Abbott Nutrition, Columbus, OH 43219, USA \\ (Submitted 22 June 2016 - Final revision received 25 November 2016 - Accepted 8 December 2016)
}

\section{Abstract}

Human milk oligosaccharides (HMO) are involved in many biological functions influencing infant health. Although HMO act locally at the intestine, recent evidence has demonstrated that HMO are partially incorporated into the systemic circulation of breast-fed infants. In the last few years, a large amount of research has been conducted using preclinical models to uncover new biological functions of HMO. The aim of this study was to evaluate the absorption and urine excretion of HMO in rats. We administered a single oral dose of the following HMO: 2 '-fucosyllactose (2'-FL), 6'-sialyllactose and lacto- $N$-neotetraose at different concentrations to adult rats. The time course of absorption of HMO into the bloodstream and their appearance in urine was studied. Our results showed that rats, similar to human infants, are able to effectively absorb a portion of HMO from the intestine into plasma and to excrete them in urine. On the basis of this, we also conducted a specific kinetic absorption study with 2'-FL, the most predominant HMO in human milk, in 9-11-d-old rat pups. Our results confirmed that a significant amount of 2'-FL was absorbed into the systemic circulation and subsequently excreted in urine during lactation in rats in a dosedepended manner. We also found basal levels of these HMO in plasma and urine of adult rats as well as rat pups as a natural result of nursing. Our data suggest that the rat may be a useful preclinical model that provides new insights into the metabolism and functions of HMO.

Key words: Human milk oligosaccharides: Rats: Human milk oligosaccharides absorption: 2'-Fucosyllactose: 6'-Sialyllactose

In mammals, the mother's milk is the unique source of energy and nutrients for the developing newborn during early life. The relative proportions of milk macronutrients differ among species in order to provide for the specific needs of their offspring $^{(1)}$. One of the major differences between human milk and milk from most mammals is the quantity and great diversity of unconjugated and complex glycans present in human milk $v$. the milk of other mammals. In human milk, the large quantities as well as the profile of human milk oligosaccharides (HMO) are unique, particularly in comparison with bovine milk, which is the basis for most infant formulae ${ }^{(2,3)}$. Over $200 \mathrm{HMO}$ have been identified in human milk ${ }^{(4-7)}$, with $2^{\prime}$-fucosyllactose (2'-FL) being the most abundant $\mathrm{HMO}$, with a concentration of up to $4.65 \mathrm{~g} / \mathrm{l}^{(8)}$. Lacto- $N$-neotetraose (LNnT) and 6 '-sialyllactose $(6 '-S L)$ are also major constituents of $\mathrm{HMO}^{(9)}$.

Accumulating evidence indicates that HMO consumption benefits the infant in multiple ways. The most well-known and referenced HMO benefit is its role as a prebiotic ${ }^{(10,11)}$. However, HMO have been associated with many diverse biological functions including protection against pathogens ${ }^{(9,10,12)}$, antiinflammatory properties ${ }^{(13,14)}$, immune system regulation ${ }^{(15,16)}$, gut development and maturation ${ }^{(17,18)}$, prevention of necrotizing enterocolitis $^{(19)}$ and enhancement of brain development ${ }^{(20,21)}$. Recent studies have identified sialylated HMO as modulators of anxious behaviour ${ }^{(22)}$, whereas fucosylated $\mathrm{HMO}$ enhance learning and memory in rodents ${ }^{(23)}$.

Although many HMO functions are dependent on the local presence of these glycans in the intestine, an increasing number of systemic health benefits have reportedly been induced by intake of HMO. Initially, the scientific community considered HMO as non-digestible, soluble fibres ${ }^{(24)}$ that passed through the gastrointestinal tract, acting as selective prebiotics for beneficial bacteria ${ }^{(25-27)}$. However, most of the ingested HMO are found to be intact and non-metabolised in infant faeces, although the amount of these HMO in faeces changes over time and also depends on the presence of saccharolytic bacteria in the host microbiota ${ }^{(28)}$. This suggests potential biological functions for HMO beyond serving as preferential bacterial substrates devoted to maintaining a healthy microbiota ${ }^{(10)}$. Studies have shown that HMO are resistant to intestinal digestion ${ }^{(29,30)}$, and Gnoth, et al. have demonstrated that HMO can be passively or actively transported across intestinal cell monolayers ${ }^{(31)}$. The presence of $\mathrm{HMO}$ in the urine of exclusively breast-fed infants $^{(32-35)}$ as well as in preterm infants ${ }^{(36)}$ suggests that

Abbreviations: 2'-FL, 2'-fucosyllactose; 3'-SL, 3'-sialyllactose; 6'-SL, 6'-sialyllactose; HMO, human milk oligosaccharides; LNnT, lacto- $N$-neotetraose. 
portions of HMO are absorbed into plasma. Moreover, recent experiments have detected the presence of some milk oligosaccharides, specially sialylated oligosaccharides, in plasma from formula-fed and partially breast-fed infants ${ }^{(37)}$, and direct evidence of $\mathrm{HMO}$ in the circulation of breast-fed infants has been established lately ${ }^{(38)}$. This confirms that, although most $\mathrm{HMO}$ are excreted in faeces, a portion of HMO is absorbed into plasma and may modulate or contribute to systemic functions.

In light of the potential importance of HMO for infant health, a significant amount of research has focused beyond the characterisation of HMO in breast milk, to discover and investigate different biological functions. Studies focused on potential HMO functions have mostly been conducted using in vitro or preclinical tools, with rodents being the most prevalent model among them ${ }^{(19,22,23,39)}$. However, little is known about HMO kinetics of absorption and their metabolic fate in animal models. In light of differences in the biology of HMO among different mammalian species ${ }^{(40)}$, some doubts may arise about whether rodent models are adequate for such research ${ }^{(41)}$. This study aimed to confirm whether those results about HMO absorption in humans are also found in rodents, confirming therefore that these preclinical models are suitable for research in the field of biological effects of HMO. Our study confirms that abundant HMO in human milk, 2'-FL, 6'-SL and $\mathrm{LNnT}^{(9)}$, are effectively absorbed into the bloodstream in adult rats. As 2'-FL is the most predominant $\mathrm{HMO}$ in human milk, a specific kinetic study of its absorption was also carried out in rat pups.

\section{Methods}

\section{Chemicals and reagents}

All reagents were of analytical grade unless specified otherwise. Water $(18.2 \mathrm{M} \Omega \mathrm{cm})$ was purified and filtered by a specific liquid chromatography (LC)-MS filter using a Milli-Q system from Millipore. Sialic acid, fucose and lactose were supplied by Sigma-Aldrich, 2'-FL and 6'-SL by Inalco (>95\% purity), and 3'-sialyllactose (3'-SL) (>95\% purity) and LNnT (>90\% purity) by Kyowa Hakko Bio Co., Ltd. Acetonitrile (MeCN) LC-MS grade, ethanol (EtOH) HPLC grade and ammonia solution $25 \%\left(\mathrm{NH}_{3}\right)$ as an eluent additive for LC-MS were obtained from Scharlab.

\section{Animals and experimental design}

Experiment in adult rats: Sprague-Dawley female rats (Charles River Laboratories) of about $300 \mathrm{~g}$ (8-10 weeks old) were used for the present study. The animals were maintained in pairs in standard cages at constant room temperature $\left(22 \pm 2^{\circ} \mathrm{C}\right)$ and $45-55 \%$ humidity under a regular $12 \mathrm{~h}$ light $-12 \mathrm{~h}$ dark schedule. Animals were fed a semi-purified nutritional regimen that followed AIN-93M guidelines (manufactured and pelletised by Abbott Laboratories). Food and water were freely available.

The experimental design was as follows: animals were randomly assigned to the experimental groups $(n 8)$ by the $\mathrm{HMO}$ and dose received. HMO were dissolved in water and administered by intragastric gavage after $12 \mathrm{~h}$ of fasting. Immediately before the intragastric gavage, a blood sample was collected from the caudal vein of each animal (time 0). After the gavage, blood samples were collected at 30, 60, 90, 120, 180, 240 and $300 \mathrm{~min}$. Blood samples were allowed to clot and then centrifuged at $5000 \mathrm{rpm}$. Serum was stored at $-30^{\circ} \mathrm{C}$ for further analysis.

Urine samples were collected when voluntary urination took place while the animal was handled.

Doses of 2'-FL administered were $0 \cdot 2,1$ and $5 \mathrm{~g} / \mathrm{kg}$ body weight (BW). Because of lower solubility, the other two oligosaccharides, 6'-SL and LNnT, were not tested at $5 \mathrm{~g} / \mathrm{kg}$ body weight. 6'-SL was tested at equimolecular doses equivalent to $0 \cdot 2,1$ and $3.75 \mathrm{~g}$ 2'-FL/kg BW, and LNnT was administered only at equimolecular doses equivalent to $0 \cdot 2$ and $1 \mathrm{~g} 2$ '-FL/ $\mathrm{kg}$ BW. A total of eight rats were used for each oligosaccharide and each dose.

Experiment in rat pups: pregnant Sprague-Dawley rats (Charles River Laboratories) were used for this experiment. After $2 \mathrm{~d}$ of delivery, all the litters were mixed and readjusted in order to retain the same number of pups for each dam ( $n 10$ pups/dam, five males and five females). On the day of the experiment, the pups were 9-11 d old. The pups were separated from dams, fasted for $4 \mathrm{~h}$ and kept warm in $28-30^{\circ} \mathrm{C}$ temperature-controlled cages. The oral bolus of 2'-FL $(0.3 \mathrm{ml})$ was administered intragastrically with a twenty-four-gauge probe at four different doses: 1, 2.5, 5 and $10 \mathrm{~g} / \mathrm{l}$. A soya formula was the vehicle as it was free of HMO and provided some energy to the pups during the experiment in order to avoid the metabolic alteration caused by prolonged fasting. At different times after the gavage (30, 60, 90, 120, 180 and $240 \mathrm{~min}$ ), the pups were killed ( $n 8$ each sampling time, four males and four females). Blood was collected, and urine was aspirated from the bladder. Plasma and urine samples were flash-frozen and stored at $-80^{\circ} \mathrm{C}$ for $\mathrm{HMO}$ determination by ultra-performance liquid chromatography-MS (UPLC-MS)/MS. An additional group of twelve pups (six males and six females) was also killed without any gavage to obtain basal levels.

The experiments were carried out in accordance with guidelines of the European Union (2010/63/UE) and the Spanish regulations (RD 53/2013, BOE-A-2013 1337) for the use of laboratory animals. All experimental protocols were also approved by the local Ethics Committee.

\section{Analysis of human milk oligosaccharides by ultra-performance liquid chromatography-MS}

Analysis of HMO in serum was carried out by UPLC-MS after sample extraction as previously described ${ }^{(42)}$. In brief, serum samples were extracted with 2-ml (2:1) (v/v) chloroformmethanol solution and $0.4 \mathrm{ml}$ of de-ionised water. After centrifugation, the upper layer was collected, and the proteins were precipitated overnight with cold ethanol. The solution was centrifuged, and the supernatant was collected, dried and then re-dissolved in $0 \cdot 1 \% \mathrm{NH}_{3}$ in water. The final extract was again centrifuged, and the supernatant was injected into the UPLC. Urine samples $(50 \mu \mathrm{l})$ were diluted in water $(150 \mu \mathrm{l}$ final volume), filtered and centrifuged in Amicon filter units (pore size: $10000 \mathrm{~K}, 14000$ rpm, $30 \mathrm{~min}$; Merck Millipore) before injection. 
Chromatographic analysis was performed using a UPLC BEH Amide column $(2.1 \times 100-\mathrm{mm}, 1.7-\mu \mathrm{m}$ particle size $)$ from Waters. The flow rate was $300 \mathrm{ml} / \mathrm{min}$, the column was maintained at $25^{\circ} \mathrm{C}$, the sample at $20^{\circ} \mathrm{C}$ and the injection volume was $1 \mu \mathrm{l}$ in partial loop mode. A gradient mobile phase consisting of $0 \cdot 1 \%(\mathrm{v} / \mathrm{v})$ ammoniacal aqueous solution (solvent $\mathrm{A}$ ) and $0 \cdot 1 \%$ (v/v) ammonia in acetonitrile (solvent B) was used. Gradient conditions were as follows: $0.0-3.0 \mathrm{~min}, 10-25 \% \mathrm{~A} ; 3.0-8.0 \mathrm{~min}$, 25-40\% A; 8.0-8.1 min, 40-10\% A; and 8.1-10.0 min $10 \% \mathrm{~A}$ to stabilise the initial conditions. The total run time was $8.1 \mathrm{~min}$, and the post-delay time for reconditioning the column with $10 \%$ A was $1.9 \mathrm{~min}$. Weak solvent was a mixture of $25-\mathrm{ml}$ water and 75-ml acetonitrile, and strong solvent was a mixture of 80-ml water and 20-ml acetonitrile. These solutions were stable for at least 1 week at room temperature.

Regarding spectrometric conditions, electrospray ionisation (ESI) was performed in negative-ion mode. The tandem mass spectrometer was operated in multiple reaction monitoring mode, and Q1 and Q3 quadrupoles were set at unit mass resolution. The mass spectrometric conditions were optimised for each compound by continuously infusing standard solutions $(0 \cdot 1-1 \cdot 0 \mathrm{mg} / \mathrm{l})$. ESI spray voltage was $3500 \mathrm{~V}$. N was used as the desolvation gas at $800 \mathrm{l} / \mathrm{min}$ and as auxiliary gas in the cone at $50 \mathrm{l} / \mathrm{min}$. The temperature of the source was $120^{\circ} \mathrm{C}$, and the desolvation temperature was $400^{\circ} \mathrm{C}$. Argon $(99.999 \%$ purity) was used as the collision gas at an approximate rate of $0.13 \mathrm{ml} / \mathrm{min}$. Selection of two specific fragmentation reactions for each compound allowed simultaneous quantification and identification in one run, ensuring a high specificity of the method. The following pairs (precursor $>$ ion) were selected to determine each compound: $(163.0>58.9)$ and $(163.0>88.9)$ for fucose; $(308.1>87.0)$ and $(308 \cdot 1>170 \cdot 1)$ for sialic acid; $(341 \cdot 1>100 \cdot 9)$ and $(341 \cdot 1>161 \cdot 1)$ for lactose; $(487.2>161 \cdot 0)$ and $(487.2>205.0)$ for $2^{\prime}-\mathrm{FL}$; $(632 \cdot 2>87 \cdot 1)$ and $(632 \cdot 2>290 \cdot 1)$ for $6 '-S L ;(632 \cdot 2>87 \cdot 1)$ and $(632 \cdot 2>264 \cdot 0)$ for 3 '-SL; $(706 \cdot 2>179 \cdot 0)$ and $(706 \cdot 2>263 \cdot 0)$ for LNnT. Quantification was performed by external calibration. The limits of detection ranged from 5 to $70 \mathrm{ng} / \mathrm{ml}$ and the limits of quantification from 20 to $200 \mathrm{ng} / \mathrm{ml}$. The inter- and intra-day variabilities were lower than $15 \%$, and the recoveries ranged from 85 to $115 \%$. For further details, see Santos-Fandila et al. ${ }^{(42)}$.

\section{Statistical analysis}

The concentrations of HMO and related sugars are given as mean values and standard deviations or standard errors.
The time courses of the 2'-FL, 6'-SL and LNnT in plasma were analysed by repeated measures two-way ANOVA. The time courses for one dose were analysed by repeated measures one-way ANOVA. When the data did not meet the assumption of homogeneity of variances, corresponding non-parametric statistics were used. Comparisons with baseline values were performed by Dunnett's or Dunn's test. Paired comparisons between baseline values and those after the gavages were performed by the Mann-Whitney test. Graph-Pad Prism 5 software was used to perform the analyses.

\section{Results}

\section{Circulating basal level of human milk oligosaccharides in adult rats}

Adult rats had low but measurable levels of several milk glycans in serum before receiving any oral bolus of HMO. However, not all the oligosaccharides tested in this study were found in all the animals. Table 1 shows the average values with standard deviations as well as the percentage of animals that presented each HMO in their serum (\% positive). For these calculations, we have used the baseline data from all the rats used in this study at 0 time, before any HMO was given to the animals ( $n$ 62). The most abundant oligosaccharide detected was 3'-SL, which was present in the majority of animals (83.9\%), followed by 6'-SL with a serum concentration five times lower than 3'-SL and present in more than half of the subjects (61.5\%). 2'-FL was only found in the serum of $13 \cdot 1 \%$ of the rats at concentrations slightly lower than 6'-SL. LNnT was not detected in any sample. For further reference, the serum HMO profile is shown in Fig. 1.

Time course of 2'-fucosyllactose, 6'-sialyllactose and lacto$\mathrm{N}$-neotetraose in plasma of adult rats after administration of a single oral dose

Although only $13 \%$ of animals showed circulating levels of 2'-FL at baseline, when 2'-FL was administered orally by gavage, it appeared in serum as early as $30 \mathrm{~min}$ in all the $2^{\prime}$-FL-dosed animals. The maximum peak of $2^{2}$-FL in serum was reached 60 min after the gavage for the lowest dose and between 90 and 120 min for higher doses of 2'-FL. After reaching the maximum peak, 2'-FL was maintained in the plasma compartment for at least the $5 \mathrm{~h}$ of this experiment, particularly when higher doses were administered (Fig. 2(a)).

Table 1. Baseline levels of human milk oligosaccharides (sialic acid, 3'-sialyllactose (3'-SL), 6'-sialyllactose (6'-SL), 2'-fucosyllactose $\left(2^{\prime}-\mathrm{FL}\right)$, lacto- $N$-neotetraose $\left.(\mathrm{LNnT})\right)$ and fucose $(\mu \mathrm{g} / \mathrm{ml})$ in serum from adult rats before receiving any oral administration* (Averages, standard deviations, percentage of relative standard deviation (\%RSD) and the percentage of animals that contained the analyte in their serum (\% positive))

\begin{tabular}{lcccccr}
\hline & \multicolumn{5}{c}{ Basal level in serum $(\mu \mathrm{g} / \mathrm{ml})$} \\
\cline { 2 - 6 } & Sialic acid & 3'-SL & 6 '-SL & 2'-FL & LNnT & Fucose \\
\hline Average & 1.140 & 0.318 & 0.0647 & 0.0454 & ND & 10.406 \\
SD & 1.0 & 0.3 & 0.1 & 0.2 & 3.0 \\
\%RSD & 90.9 & 107.0 & 154.7 & 340.9 & 28.8 \\
\% Positive & 100 & 83.9 & 61.5 & 13.1 & 0 & 100 \\
\hline
\end{tabular}

* Samples were analysed by ultra-performance liquid chromatography-MS/MS. 


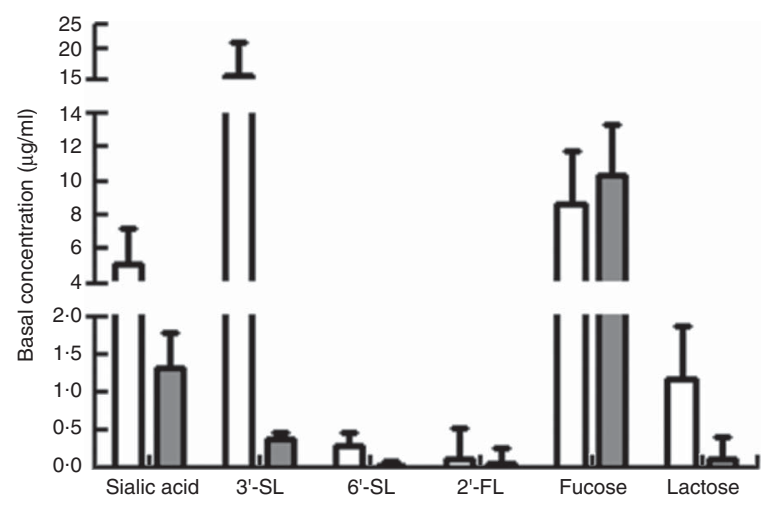

Fig. 1. Basal circulating human milk oligosaccharides and related sugars (sialic acid, 3'-sialyllactose (3'-SL), 6'-sialyllactose (6'-SL), 2'-fucosyllactose (2'-FL), fucose and lactose) in serum from pups $(\square)$ and adult rats ( $\square$ ). Lacto- $N$-neotetraose was not detected in any sample at baseline.

The parent sugars of 2'-FL, lactose and fucose, increased in plasma after the administration of $2^{\prime}-\mathrm{FL}$. This was particularly noteworthy for lactose and in animals administered the higher dose of 2'-FL, as shown in Fig. 3(e). Another interesting aspect is that not only lactose concentration was increased during the time course of the experiment but also the number of subjects presenting lactose in serum. Thus, only $12 \%$ of animals had detectable levels of lactose in serum at baseline, and this percentage increased over time reaching $100 \%$ at $300 \mathrm{~min}$ after the gavage. Likewise, circulating levels of sialic acid and 3'-SL were affected by the process of absorption of 2'-FL after the gavage (Fig. 3(a) and (b)). The overall ANOVA analysis did not show a significant effect $(P=0 \cdot 1848$ and $P=0 \cdot 1650$ for sialic acid and 3'SL, respectively); however, individual comparisons by paired $t$ test showed that there were differences between basal levels and levels at 30,60 and 90 min for sialic acid and $60 \mathrm{~min}$ for 3'-SL. Those trends could not be explained by the presence of these compounds in the source of 2'-FL used in the gavages, because it had purity higher than $95 \%$ and it was free of sialic acid or any other HMO (Table 2).

With regard to animals receiving 6'-SL, about $60 \%$ of rats exhibited naturally circulating 6'-SL at baseline. After the gavages, 6'-SL was quickly absorbed into the circulation, showing significant amounts of 6'-SL at $60 \mathrm{~min}$ after the oral bolus was administered. Serum 6'-SL levels did not decrease within the $300 \mathrm{~min}$ of the experiment (Fig. 2(b)). Sialic acid steadily increased over time after the gavage of 6'-SL (Fig. 4(a)). No differences were found in levels of other HMO and sugars.

As commented above, LNnT was not detectable in serum in any subject at baseline. However, LNnT appeared in serum 30 min after gavage with LNnT. At the lowest dose, the peak was reached at $60 \mathrm{~min}$, and the serum LNnT level began to decrease slowly in plasma at $90 \mathrm{~min}$. The absorption profile of LNnT after the gavage with the higher dose was slightly different: a first peak appeared as early as $30 \mathrm{~min}$ followed by a plateau phase of $90 \mathrm{~min}$, after which the level of serum LNnT reached the maximum level $(180 \mathrm{~min})$ and began to decline (Fig. 2(c)). The absorption of LNnT did not significantly affect the concentration of the other analysed compounds in the
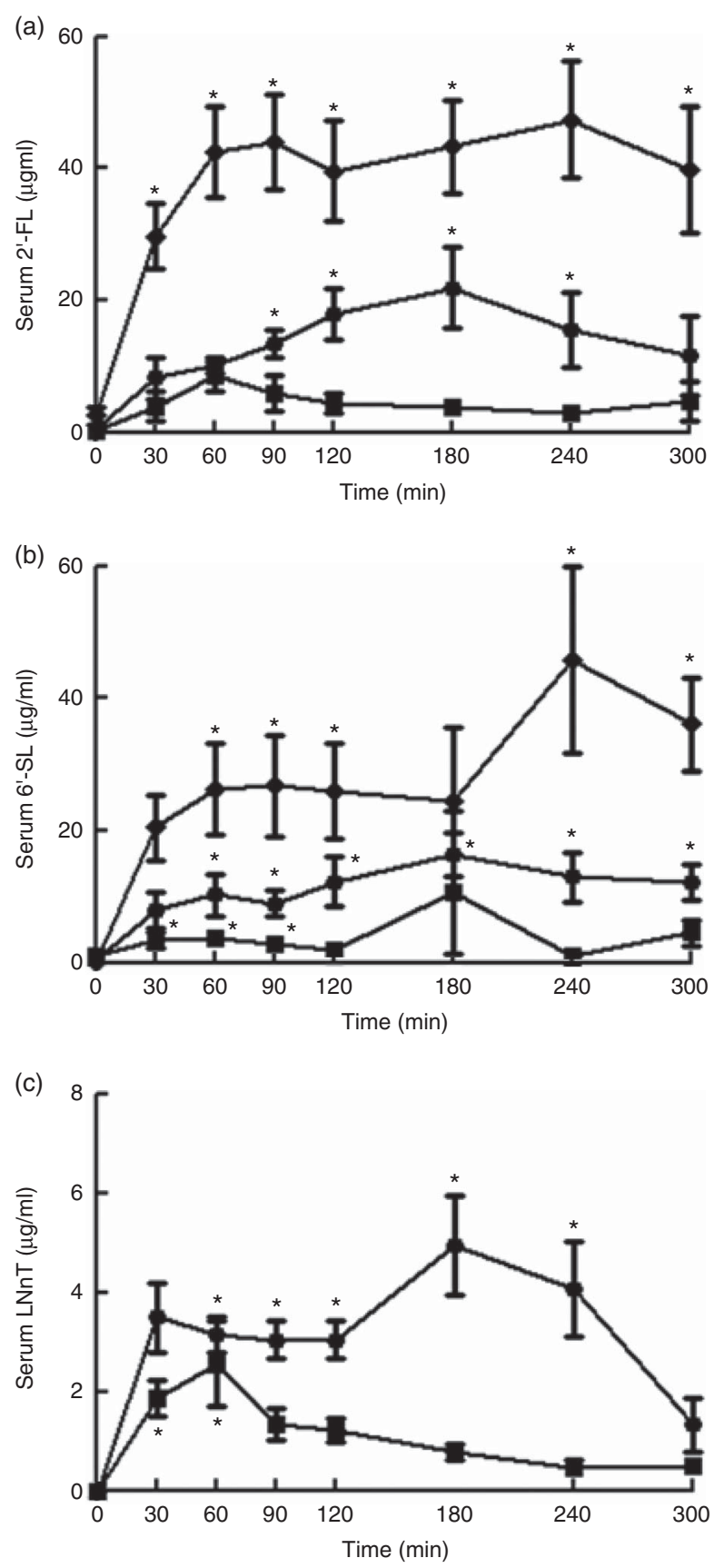

Fig. 2. (a) Time course of $2^{\prime}$-fucosyllactose $\left(2^{\prime}-F L\right)$ in serum after an oral gavage of 2 -FL. (b) Time course of 6'-sialyllactose (6'-SL) in serum after an oral gavage of 6'-SL. (c) Time course of lacto- $N$-neotetraose (LNnT) in serum after an oral gavage of LNnT. The doses for 2'-FL gavages were 0.2 (dose $A,--$ ), 1 (dose $\mathrm{B},-$ ) and 5 (dose $\mathrm{C},-\mathrm{g} / \mathrm{kg}$ body weight. The other two oligosaccharides, 6'-SL and LNnT, were given in equimolecular doses to those of 2'-FL, except in the highest doses, because of technical issues based on the solubility properties of 6'-SL and LNnT. Thus, 6'-SL was tested at equimolecular doses equivalent to 0.2 (dose A), 1 (dose B) and 3.75 (dose C) g 2'-FL/kg BW; LNnT was given only at equimolecular doses equivalent to 0.2 (dose $A$ ) and 1 (dose B) g 2'-FL/kg BW. * $P<0.05$ v. time 0 by Dunnett's or Dunn's multiple comparison test.

bloodstream, with the exception of lactose. When the higher dose of LNnT was administered, lactose increased 30 min after the LNnT bolus (data not shown). 

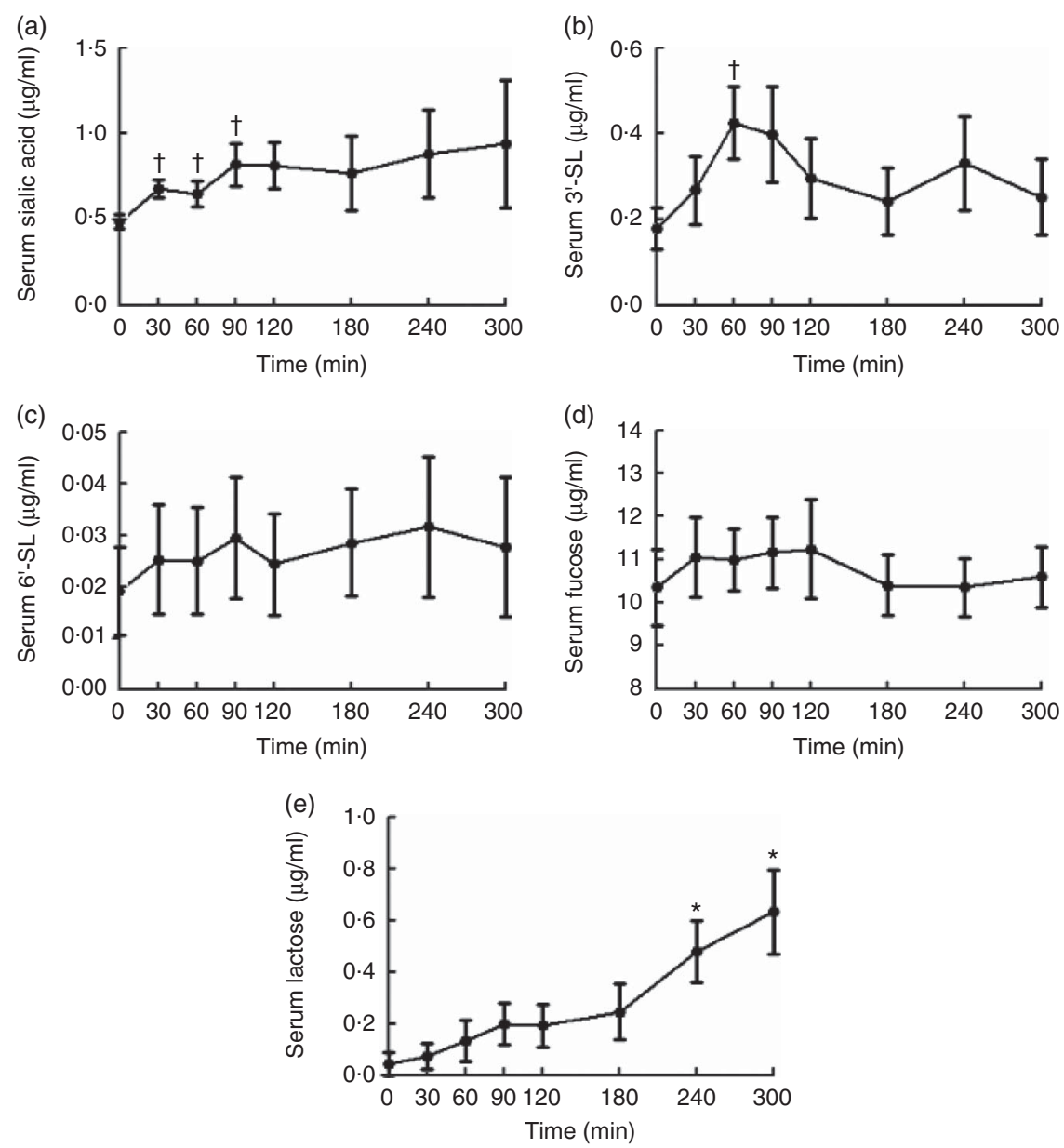

Fig. 3. Time course of sialic acid (a), $3^{\prime}$-sialyllactose ( $\left.3^{\prime}-\mathrm{SL}\right)(\mathrm{b}), 6^{\prime}$-sialyllactose (6'-SL) (c), fucose (d) and lactose (e) in serum after an oral gavage of $2^{\prime}$-fucosyllactose $\left(2^{\prime}-\mathrm{FL}\right)\left(5 \mathrm{~g} 2^{\prime}-\mathrm{FL} / \mathrm{kg}\right.$ body weight). ${ }^{*} P<0.05 \mathrm{v}$. time 0 by Dunnett's multiple comparison test. $\dagger P<0.05 \mathrm{v}$. time 0 by paired $t$ test.

Table 2. Serum lactose levels $(\mu \mathrm{g} / \mathrm{ml})$ from adult rats administered $2^{\prime}$-fucosyllactose $(5 \mathrm{~g} / \mathrm{kg}$ body weight) before receiving any oral administration*

(Averages, standard deviations, percentage of relative standard deviation (\%RSD) and the percentage of animals that contained the analyte in their serum (\% positive))

\begin{tabular}{lccccccccc}
\hline & \multicolumn{8}{c}{ Serum lactose level $(\mu \mathrm{g} / \mathrm{ml})$} \\
\cline { 2 - 9 } & T0 & T30 & T60 & T90 & T120 & T180 & T240 & T300 \\
\hline Average & 0.043 & 0.073 & 0.134 & 0.196 & 0.192 & 0.256 & 0.478 & 0.633 \\
SD & 0.1 & 0.1 & 0.2 & 0.2 & 0.2 & 0.3 & 0.3 & 0.4 \\
\%RSD & $282 \cdot 8$ & 201.4 & 170.2 & 114.9 & $122 \cdot 0$ & 116.2 & 67.0 & 67.8 \\
\% Positive & 12 & 25 & 38 & 50 & 50 & 57 & 86 & 100 \\
\hline
\end{tabular}

* Samples were analysed by ultra-performance liquid chromatography-MS/MS.

Urinary excretion of human milk oligosaccharides in adult rats after administration of a single oral dose

A urinalysis provides information about accumulated concentrations of circulating compounds over time. Despite being unable to collect samples from all the subjects at all the sampling times and that the resulting data were highly variable, we found that excretion of all three HMO began between 90 and 120 min after the gavage, especially at the highest dose (Fig. 5).
Interestingly, the other HMO and related sugars, which were not administered to the animals, were also found in urine at various concentrations across the experimental time period. Fig. 6-8 show comparisons of these compounds between the baseline $(T=0)$ and the time when the maximum peak was reached for 2'-FL, 6'-SL and LNnT, respectively, and for any given dose after the gavage. In fact, the concentrations of any HMO and sugars in urine were increased with respect to baseline values. The increase was more remarkable for the 

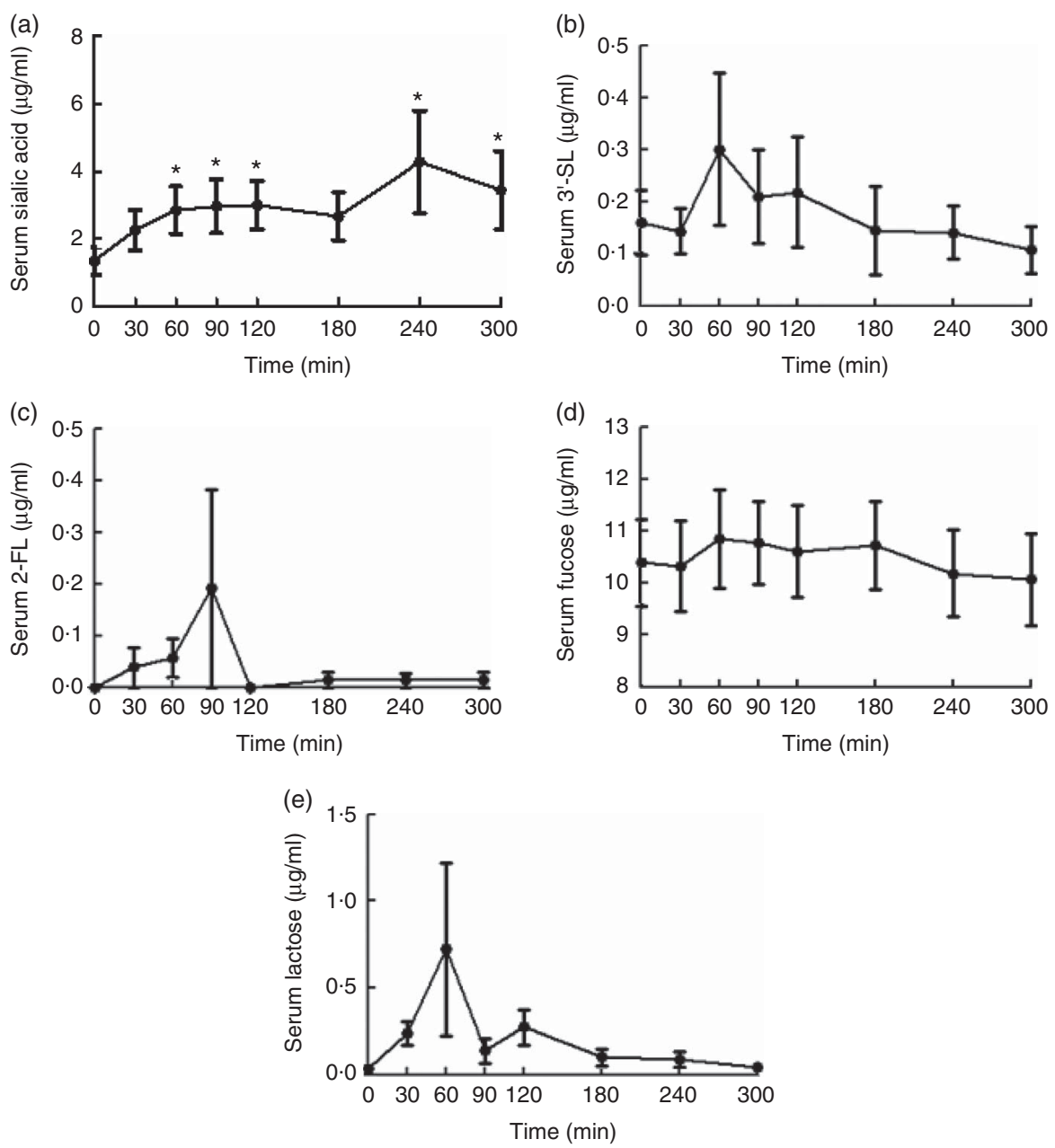

Fig. 4. Time course of sialic acid (a), $3^{\prime}$-sialyllactose (3'-SL) (b), $2^{\prime}$-fucosyllactose (2'-FL) (c), fucose (d) and lactose (e) in serum after an oral gavage of $6^{\prime}$-sialyllactose (equimolar amount to $3.75 \mathrm{~g} \mathrm{2'-FL/kg} \mathrm{body} \mathrm{weight).}{ }^{*} P<0.05 \mathrm{v}$. time 0 by Dunn's multiple comparison test.

parent sugars, lactose and fucose, after 2'-FL gavage and for sialic acid after the 6'-SL gavage, but it also happened for other non-structure-related compounds.

Time course of 2'-fucosyllactose in plasma of rat pups after administration of a single oral dose

Fig. 1 displays the basal amounts of sialic acid, 3'-SL, 6'-SL, 2'-FL as well as the carbohydrates fucose and lactose in pup sera after $4 \mathrm{~h}$ of fasting. Fig. 9(a) shows the absorption of $2^{\prime}-\mathrm{FL}$ from the intestine to the plasma compartment during $4 \mathrm{~h}$ after the gavages. There was no sex effect, and thus the data were pooled. Absorption curves were clearly dose dependent, although the absorption rate was much slower in the lower doses compared with the higher doses, where the slopes of the curves (doses 5 and $10 \mathrm{~g} / \mathrm{l}$ ) were more remarkable. The amount of circulating 2'-FL progressively increased with time, and did not reach a peak or saturation within the 4-h sampling period.

Unlike adult animals, there were no significant changes during the time course of the experiment in any other HMO and associated compounds with respect to baseline (data not shown) with the exception of fucose. Interestingly, when a gavage of 2'-FL was administered to the pups, fucose increased proportionally to the concentration of 2'-FL with a profile similar to 2'-FL, also reaching maximum absorption at $180 \mathrm{~min}$ (Fig. 9(c)). In addition, basal lactose serum concentrations were ten times higher in pups than in adult rats (Fig. 1). No significant changes were noted in plasma lactose concentration in pups after the gavages with 2 '-FL. This is in contrast to adult rats, whose serum lactose level progressively increased after the gavage with 2'-FL.

\section{Excretion of human milk oligosaccharides in urine of rat pups after administration of a single oral dose of 2'-fucosyllactose}

The time course of 2'-FL and related compounds in urine was quite similar to serum samples. The excretion of 2'-FL was dose dependent and exhibited a progressive and constant increase over time (Fig. 9(c)). Sialic acid, 3'-SL and 6'-SL were also detected in pup urine, both at baseline and after the gavages with 2'-FL. As in serum samples, these HMO and sugars, with 

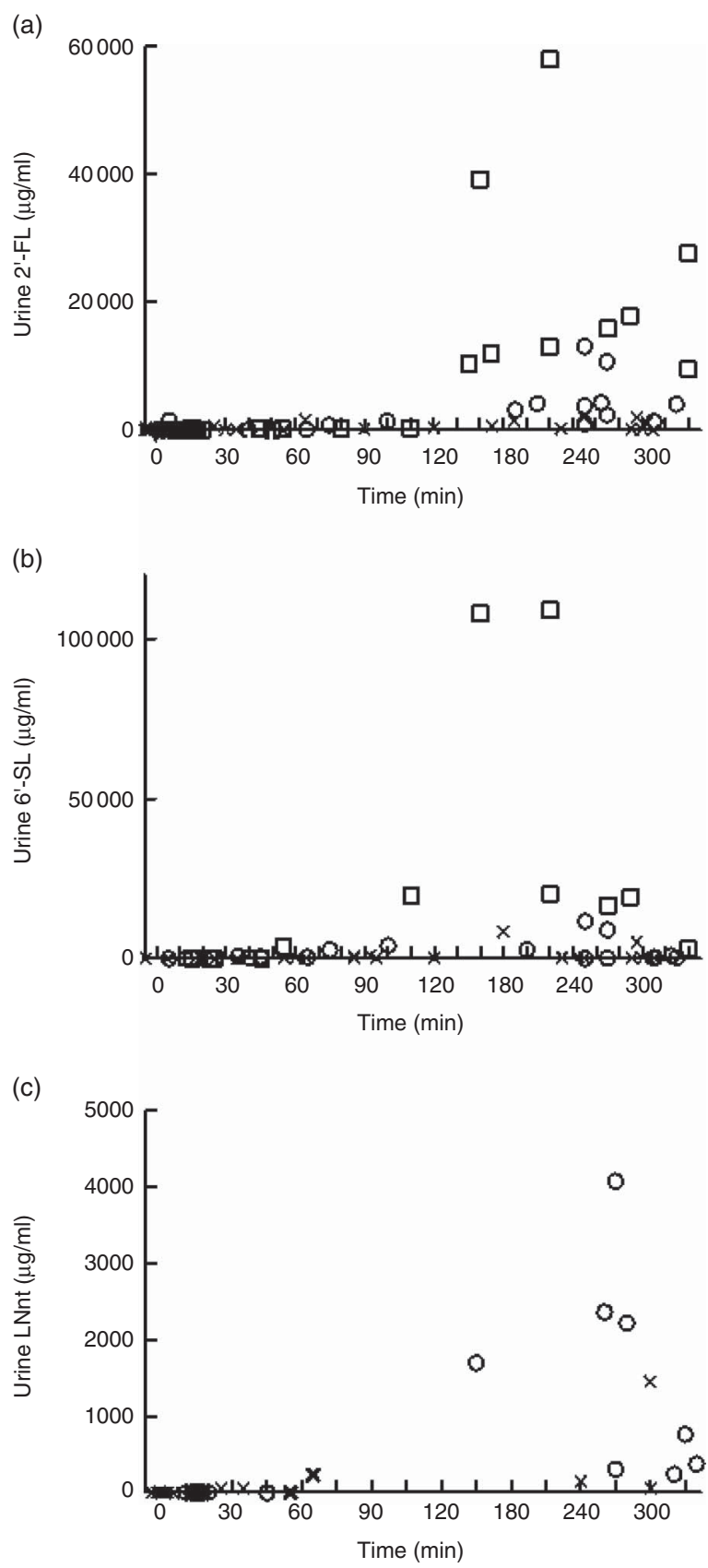

Fig. 5. (a) Time course of $2^{\prime}$-fucosyllactose $\left(2^{\prime}-\mathrm{FL}\right)$ in urine after an oral gavage of 2'-FL. (b) Time course of 6'-sialyllactose (6'-SL) in urine after an oral gavage of 6'-SL. (c) Time course of lacto- $N$-neotetraose $(\mathrm{LNnT})$ in urine after an oral gavage of $L N n T$. The doses for 2'-FL gavages were $0.2(X), 1(O)$ and $5(\square) \mathrm{g} / \mathrm{kg}$ body weight (BW). The other two oligosaccharides, 6'-SL and LNnT, were given in equimolecular doses to those of 2'-FL, except in the highest doses, because of lower solubility of 6'-SL and LNnT. Thus, 6'-SL was tested at equimolecular doses equivalent to $0.2(X), 1(O)$ and $3.75(\square)$ g 2'-FL/kg BW; $\mathrm{LNnT}$ was given only at equimolecular doses equivalent to $0.2(X)$ and $1(\mathrm{O}) \mathrm{g}$ 2'-FL/kg BW.

the exception of fucose, did not change over the $4 \mathrm{~h}$ after the administration of $2^{\prime}$-FL, regardless of dose or sex (data not shown). Fucose increased in urine over time in a dosedependent manner in accordance with the pattern in serum (Fig. 9(d)). Interestingly, LNnT, which was not detected in the plasma compartment, was measured in pup urine at low levels $(0.5-2.5 \mu \mathrm{g} / \mathrm{ml})$. LNnT was detected in urine samples at baseline as well as in all the other sampling times, with little variation in LNnT urine concentration over time (data not shown). The presence of LNnT in urine supports the presence of LNnT in the circulation, although it was not detected in serum.

\section{Discussion}

HMO were previously thought to influence infant health exclusively by local interaction at the intestine, either modulating host cells or supporting commensal bacteria. Now there is both indirect ${ }^{(32-36)}$ and direct evidence $e^{(37,38)}$ that a portion of HMO is absorbed from the intestine into the systemic circulation of breast-fed infants. However, clinical interventions pose ethical and practical limitations for the study of dosing or mechanisms of action, and preclinical models are frequently used for this purpose. In this study, we administered HMO orally at different doses to adults and rat pups, with their absorption into plasma and excretion in urine measured. Rats were given both HMO that are natural to this species, such as 6'-SL, as well as HMO that are absent or present at low concentrations in rat milk, such as $\mathrm{LNnT}$ and $2^{\prime}-\mathrm{FL}^{(3,40)}$. It is worth noting that all three were absorbed from the intestine into the bloodstream. The passage of HMO into plasma was clearly dose dependent for all the HMO administered. In adults, HMO appeared in plasma a short time after the oral bolus and reached peak absorption about $1-2 \mathrm{~h}$ after the gavage. Plasma data were in agreement with data from urine samples. Clearance of circulating HMO to urine began about $1 \mathrm{~h}$ after the gavage, reaching maximum peaks from the 2nd hour onwards. Although $\mathrm{HMO}$ were effectively cleared from circulation in a relatively short time, they remained in the bloodstream for several hours. In all the groups, plasma HMO levels were still elevated at the end of the study ( $5 \mathrm{~h}$ after the gavage) as compared with baseline.

There is limited information about HMO absorption in the published literature, let alone the kinetics of HMO absorption. For example, an interesting study performed in neonatal rats measured the presence of HMO in plasma and urine of rat pups after feeding them a mix of HMO to mimic the human milk profile $^{(41)}$. In this study, the only HMO detected in serum was 3'-SL, despite the fact that it was a minor component of the HMO-containing formula that was fed to the animals. Only a small amount of 2'-FL was detected in urine, even though 2'-FL was the most abundant HMO in the study formula. The authors concluded that this was an indication of the existence of a specific absorption mechanism for $3^{\prime}-\mathrm{SL}^{(41)}$. Our study does not support their hypothesis as we measured 2'-FL in serum in a dose-dependent manner using doses of 2'-FL within the range administered in Jantscher-Krenn's study. However, there are important differences in the experimental designs between the studies, with the age of rats being an especially relevant key factor. As $3^{\prime}$-SL is the most abundant HMO in rat milk ${ }^{(40)}$, it is possible that the biology of such young animals may have modified the response. In our study, we used 9-11-d-old rat pups and only administered 2'-FL. 
(a)

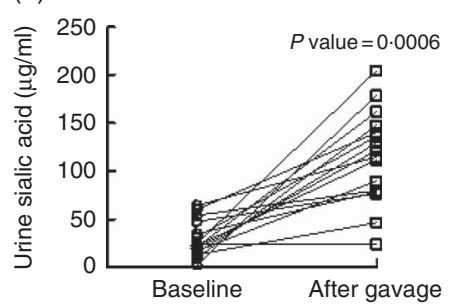

(b)

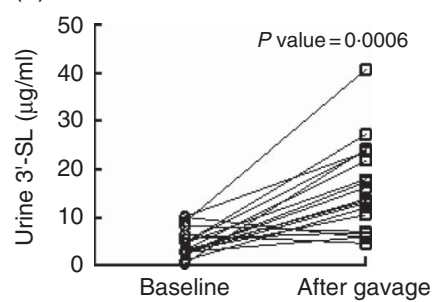

(c)

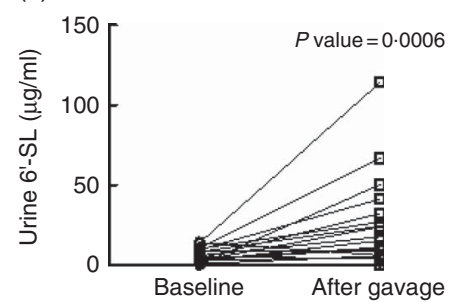

(d)

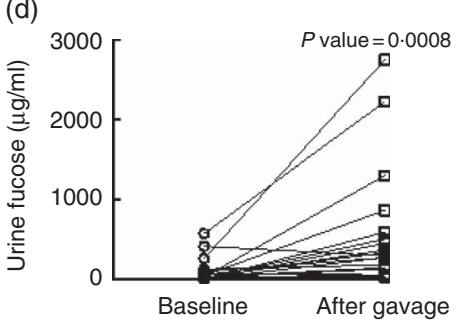

(e)

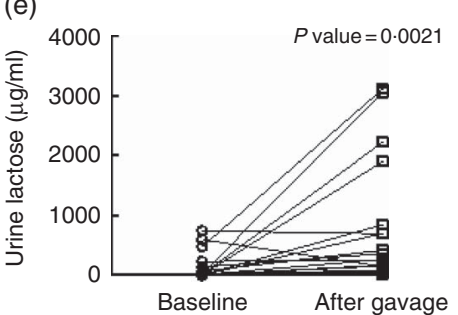

Fig. 6. Comparison of the concentrations of sialic acid (a), 3'-sialyllactose (3'-SL) (b), 6'-sialyllactose (6'-SL) (c), fucose (d) and lactose (e) in urine at baseline (T0) and at the maximum peak after an oral gavage with 2'-fucosyllactose (all doses).

(a)

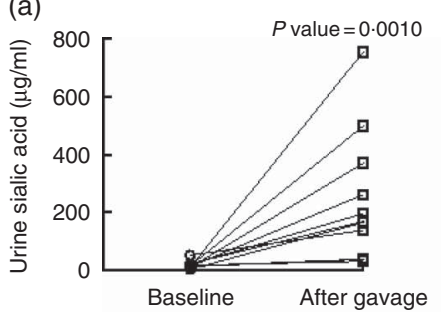

(b)

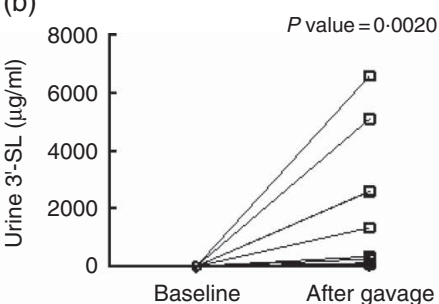

(c)

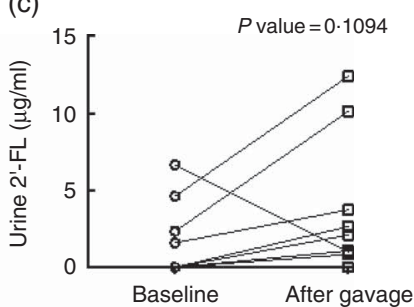

(d)

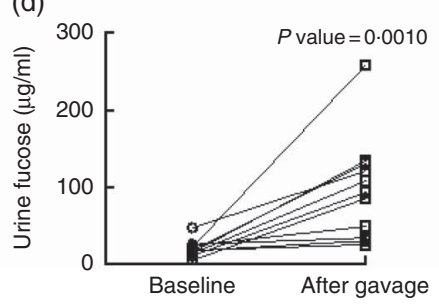

(e)

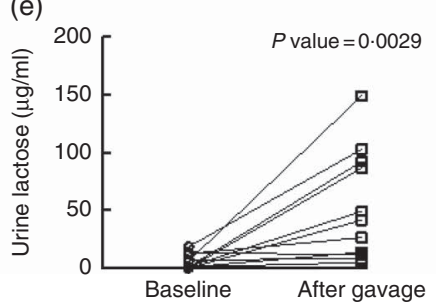

Fig. 7. Comparison of the concentrations of sialic acid (a), $3^{\prime}$-sialyllactose (3'-SL) (b), $2^{\prime}$-fucosyllactose (2'-FL) (c), fucose (d) and lactose (e) in urine at baseline (T0) and at the maximum peak after an oral gavage with 6'-sialyllactose (all doses).

Nevertheless, Jantscher-Krenn et al. ${ }^{(41)}$ found that when they analysed the oligosaccharides in different intestinal segments in neonatal pups, the initial relative abundance of 2'-FL in the HMO mix was markedly decreased across time, suggesting either local consumption by the resident bacteria or metabolism of this oligosaccharide into different derivatives that are absorbed into circulation thereafter. It is likely that oral 2'-FL has more than one fate in the organism, and that several alternatives may coexist simultaneously. These include the following: (1) the well-known and generally accepted prebiotic properties of 2 '-FL and other $\mathrm{HMO}^{(26)}$; (2) the absorption and passage of $\mathrm{HMO}$ into the systemic circulation as we have demonstrated in this study; and finally (3) HMO, especially 2'-FL, may be partially metabolised in the intestine of rat pups as suggested by several authors such as Jantscher-Krenn et al. ${ }^{(41)}$.
Dotz et al. ${ }^{(29)}$ recently published an extensive study with new insights about the metabolism of neutral HMO in exclusively breast-fed infants as well as indications for multiple factors influencing the metabolic fate of HMO. In that study, they described the presence of novel HMO metabolites in urine and faeces of breast-fed infants. Those novel HMO derivatives were identified as acetylated HMO and other HMO-like structures, produced by the infants or by their gut microbiota. Furthermore, they found secretor- or Lewis-specific HMO in the faeces/urine of infants fed non-secretor or Lewis-negative milk, which suggested a corresponding modification of HMO in the infant. The concept that HMO may be partially processed in the intestine is also supported by our data. Although the purity of the 2'-FL administered to the rats was higher than $95 \%$, the amount of fucose found in the bloodstream as well as in the 
(a)
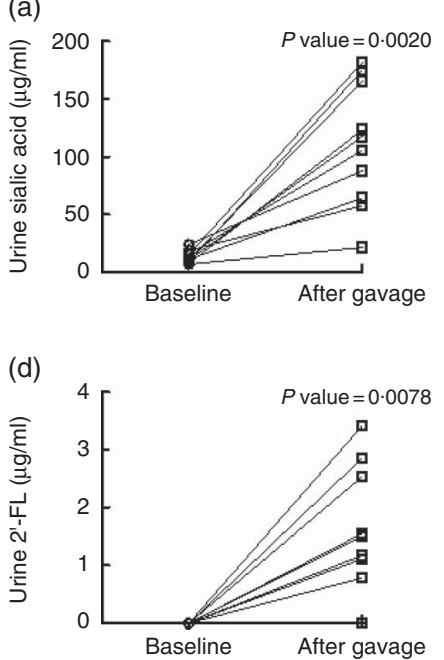

(b)

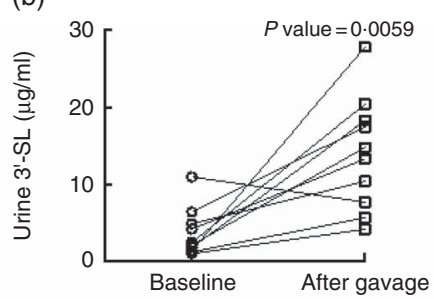

(e)

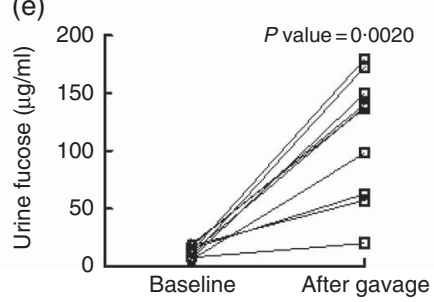

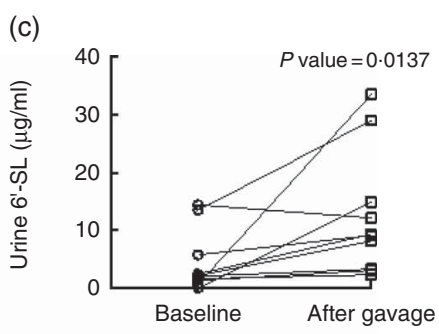

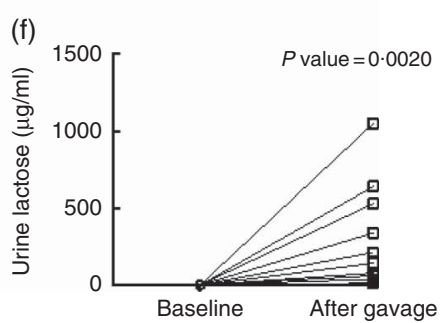

Fig. 8. Comparison of the concentrations of sialic acid (a), 3'-sialyllactose (3'-SL) (b), 6'-sialyllactose (6'-SL) (c), 2'-fucosyllactose (2'-FL) (d), fucose (e) and lactose (f) in urine at baseline (TO) and at the maximum peak after an oral gavage with lacto- $N$-neotetraose (all doses).
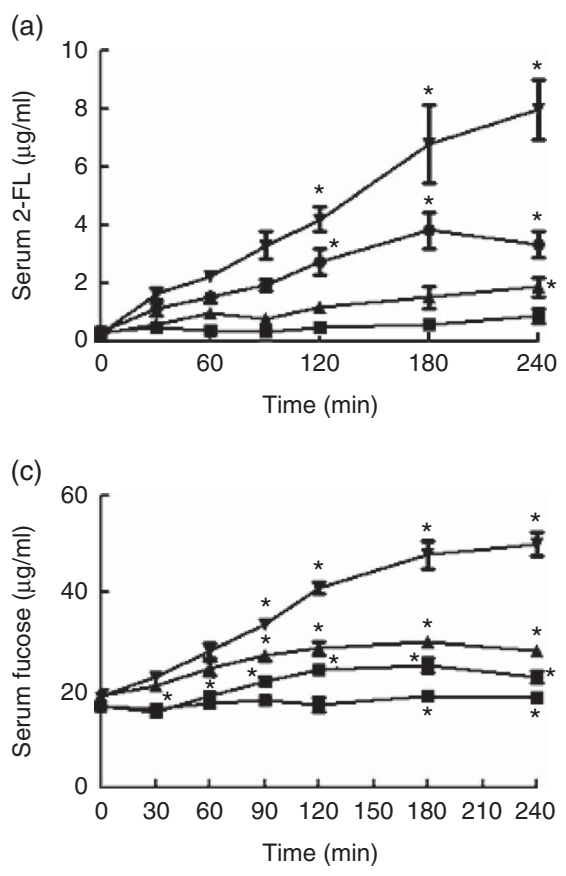

(b)

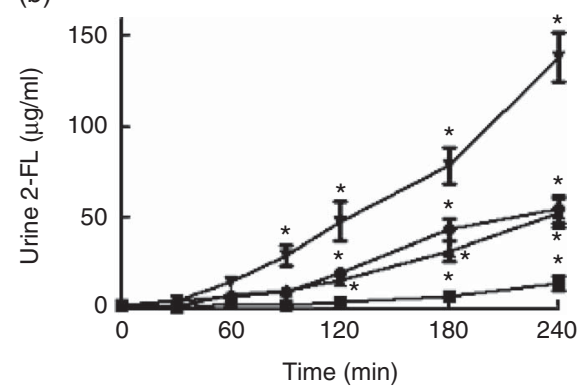

(d)

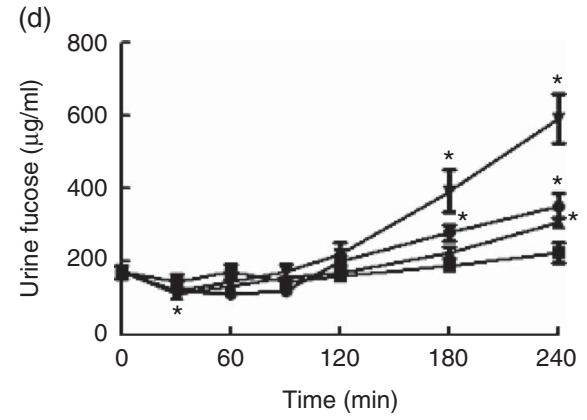

Fig. 9. The time course of 2'-fucosyllactose (2'-FL) in serum (a) and urine (b), and the time course of fucose in serum (c) and urine (d) of 9-11-d-old rats receiving an oral gavage of 2'-FL at doses of $1(\square), 2.5(\mathbf{\Lambda}), 5(\mathbf{O})$ and $10(\boldsymbol{\nabla}) \mathrm{g} / \mathrm{l}$. ${ }^{*} P<0.05 \mathrm{v}$. time 0 by Dunnet's multiple comparison test.

urine of lactating rats was in parallel with the concentration of 2'-FL in these two body compartments. This suggests that a portion of 2'-FL was metabolised to a certain extent in the intestine, generating free fucose that was rapidly absorbed into the bloodstream. However, the amount of lactose in pup serum did not significantly change with time, as fucose and 2'-FL did, and the opposite happened in adult animals. This result may be explained by the fact that intestinal lactases are highly expressed in lactating animals, and thus all the lactose potentially generated by HMO degradation was rapidly digested into single monosaccharides. In contrast, the decrease in intestinal lactase in the gut of adult animals may explain the increase in serum lactose after 2'-FL administration. However, we do not have a feasible explanation for the flat profile of serum fucose found in adult rats. It is possible that as fucose has been postulated as a key molecule for the maintenance of a balanced microbiome ${ }^{(43)}$ all the fucose released from ingested HMO might be rapidly used to satisfy the needs of the microbiome. In addition, it is worthy to mention that, once we detected these changes in fucose and lactose contents in rat plasma after the HMO gavages, we analysed all the HMO used in our study to confirm the relative presence or absence of other contaminant sugars such 
as fucose, lactose or sialic acid in order to rule out that our HMO sources were also providing significant amounts of these carbohydrates. Our results confirmed that the HMO sources used in this study contained very little or almost none of these sugars (data not shown). In fact, regarding 2'-FL, we were not able to detect traces of fucose, sialic acid or sialylated HMO, detecting only lactose at $1.5 \%$. Likewise, the purity of our source of 6'-SL was very high, although we detected the presence of a contaminant $-5 \%$ sialic acid. However, we were not able to detect any other $\mathrm{HMO}$, fucose or even lactose, in our 6'-SL stock. LNnT was the least pure HMO we used (>90\%), which was also absent of fucose, 2'-FL or sialylated HMO, and had lactose and sialic acid in very small amounts (1.6 and $0.3 \%$, respectively). On the basis of this, we consider that the origin of fucose and lactose found in the plasma compartment of rats was from HMO fermentation by intestinal microbiota, as previously mentioned $^{(28,29,36)}$.

Data regarding distribution of HMO throughout the body are scarce. Very little is known regarding the fate of HMO beyond the digestive system, with many questions remaining to be answered. We do not know exactly how HMO reach the bloodstream, where they go (target organs, and cells) and how they are excreted into urine. Further studies are needed to elucidate the fate of systemic HMO as well as the mechanism of HMO transport across biological barriers.

Our present study confirms a preliminary observation made by our group when we set up the methodology to measure $\mathrm{HMO}^{(42)}$ We confirmed that rats showed measurable levels of some HMO (3'-SL, 6'-SL and 2'-FL) in serum before being administered HMO gavages. This may be logical for lactating rats, especially considering that $3^{\prime}$-SL is the major HMO in rat milk ${ }^{(40)}$. In our experiment, rat pups were fasted for $4 \mathrm{~h}$, and Jantscher-Krenn et al. ${ }^{(41)}$ described the presence of HMO in the distal part of the intestine even after $8 \mathrm{~h}$. However, this does not explain the presence of fucosylated $\mathrm{HMO}$, which are rare in rat milk, nor does it explain the presence of HMO in serum of adult female rats. Adult rats expressed low but measurable levels of some HMO in plasma. Not all the animals expressed all the analysed HMO. LNnT was not detected in any sample, whereas sialylated HMO, especially 3'-SL, were found in most animals, and 2'-FL was detected only in about $15 \%$ of the subjects. The presence of $\mathrm{HMO}$ in the circulation of adult animals generates new questions: are HMO also present in the circulation of male adult rats? What is the biological purpose of this phenomenon? What biological functions are they involved in? Where are they synthesised? They may be released from the glycocalyx of circulating cells or the glycocalyx of interior blood vessels. It is also possible that they come from digestion of gut mucus by bacteria in the gut and are transported into the circulation. Studies to specifically address these questions are needed.

A second unexpected finding was that oral administration of an individual HMO, which was partially absorbed into the plasma and subsequently cleared to urine impacted the concentration of other HMO in serum and in urine. This previously unknown phenomenon was observed only in adult animals and not in pups, and was particularly noticeable in urine. It happened with each of the three HMO administered to the animals (2'-FL, 6'-SL and LNnT), and was more evident when the higher doses were administered. These changes in circulating HMO were more detectable in urine than in serum and may be a result of the urinary system's role as a biological concentrator of plasma solutes. The biological relevance of this finding is currently unknown. To our knowledge, this is the first time that this phenomenon has been reported. Further studies into the mechanisms of HMO absorption and metabolism at different ages are needed to understand the differences observed.

\section{Acknowledgements}

The authors thank Gera Duska-McEwen, MS for editorial review of the manuscript.

This research received no specific grant from any funding agency or from commercial or not-for-profit sectors. The funder, Abbott Nutrition, did not provide any specific grant/ funds to support the present study other than the regular annual budget for research activities as well as authors' salaries. Abbott Nutrition, as an institution, had no role in the design, analysis or writing of this article.

Regarding the authorship, all the authors, E. V., A. S.-F., R. B., R. R. and M. R., have actively participated in writing the manuscript. E. V., M. R., R. R. and R. B. were involved in the study conception as well as in the experimental design. E. V. and A. S.-F. were involved in technical work such as animal work and analytical measurements. E. V., A. S.-F., M. R., R. B. and R. R. were also involved in data review and data analysis. All authors have contributed to the study, and the final version of the manuscript has been read and approved by all named authors.

The authors are currently working for Abbott Nutrition, which is a company that manufactures infant formulae. Other than that, there are no known conflicts of interest associated with this publication.

\section{References}

1. Jensen RG (1995) Handbook of Milk Composition. San Diego, CA: Academic Press.

2. Tao N, DePeters EJ, Freeman S, et al. (2008) Bovine milk glycome. J Dairy Sci $\mathbf{9 1}, 3768-3778$.

3. Urashima T, Taufik E, Fukuda K, et al. (2013) Recent advances in studies on milk oligosaccharides of cows and other domestic farm animals. Biosci Biotechnol Biochem $\mathbf{7 7}$, 455-466.

4. Wu S, Grimm R, German JB, et al. (2011) Annotation and structural analysis of sialylated human milk oligosaccharides. J Proteome Res 10, 856-868.

5. Wu S, Tao N, German JB, et al. (2010) Development of an annotated library of neutral human milk oligosaccharides. J Proteome Res 9, 4138-4151.

6. Marino K, Lane JA, Abrahams JL, et al. (2011) Method for milk oligosaccharide profiling by 2 -aminobenzamide labeling and hydrophilic interaction chromatography. Glycobiology 21, $1317-1330$.

7. Ruhaak LR \& Lebrilla CB (2012) Advances in analysis of human milk oligosaccharides. Adv Nutr 3, 406S-414S.

8. Erney RM, Malone WT, Skelding MB, et al. (2000) Variability of human milk neutral oligosaccharides in a diverse population. J Pediatr Gastroenterol Nutr 30, 181-192. 
9. Kunz C, Rudloff S, Baier W, et al. (2000) Oligosaccharides in human milk: structural, functional, and metabolic aspects. Annu Rev Nutr 20, 699-722.

10. Bode L (2012) Human milk oligosaccharides: every baby needs a sugar mama. Glycobiology 22, 1147-1162.

11. German JB, Freeman SL, Lebrilla CB, et al. (2008) Human milk oligosaccharides: evolution, structures and bioselectivity as substrates for intestinal bacteria. Nestle Nutr Workshop Ser Pediatr Program 62, 205-218.

12. Newburg DS, Ruiz-Palacios GM \& Morrow AL (2005) Human milk glycans protect infants against enteric pathogens. Annu Rev Nutr 25, 37-58.

13. Jantscher-Krenn E \& Bode L (2012) Human milk oligosaccharides and their potential benefits for the breast-fed neonate. Minerva Pediatr 64, 83-99.

14. Kunz C \& Rudloff S (2008) Potential anti-inflammatory and anti-infectious effects of human milk oligosaccharides. Adv Exp Med Biol 606, 455-465.

15. Klein N, Schwertmann A, Peters M, et al. (2000) Immunomodulatory effects of breast milk oligosaccharides. Adv Exp Med Biol 478, 251-259.

16. Newburg DS (2009) Neonatal protection by an innate immune system of human milk consisting of oligosaccharides and glycans. J Anim Sci 87, 26-34.

17. Kuntz S, Kunz C \& Rudloff S (2009) Oligosaccharides from human milk induce growth arrest via G2/M by influencing growth-related cell cycle genes in intestinal epithelial cells. Br J Nutr 101, 1306-1315.

18. Kuntz S, Rudloff S \& Kunz C (2008) Oligosaccharides from human milk influence growth-related characteristics of intestinally transformed and non-transformed intestinal cells. Br J Nutr 99, 462-471.

19. Jantscher-Krenn E, Zherebtsov M, Nissan C, et al. (2012) The human milk oligosaccharide disialyllacto-N-tetraose prevents necrotising enterocolitis in neonatal rats. Gut 61, 1417-1425.

20. Wang B (2009) Sialic acid is an essential nutrient for brain development and cognition. Annu Rev Nutr 29, 177-222.

21. Wang B (2012) Molecular mechanism underlying sialic acid as an essential nutrient for brain development and cognition. Adv Nutr 3, 465S-472S

22. Tarr AJ, Galley JD, Fisher SE, et al. (2015) The prebiotics 3'Sialyllactose and 6'Sialyllactose diminish stressor-induced anxiety-like behavior and colonic microbiota alterations: evidence for effects on the gut-brain axis. Brain Behav Immun 50, 166-177.

23. Vazquez E, Barranco A, Ramirez M, et al. (2015) Effects of a human milk oligosaccharide, 2'-fucosyllactose, on hippocampal long-term potentiation and learning capabilities in rodents. J Nutr Biochem 26, 455-465.

24. Kunz C (2012) Historical aspects of human milk oligosaccharides. Adv Nutr 3, 430S-439S.

25. Asakuma S, Hatakeyama E, Urashima T, et al. (2011) Physiology of consumption of human milk oligosaccharides by infant gut-associated bifidobacteria. J Biol Chem 286, 34583-34592.

26. Marcobal A, Barboza M, Froehlich JW, et al. (2010) Consumption of human milk oligosaccharides by gut-related microbes. J Agric Food Chem 58, 5334-5340.
27. Zivkovic AM, German JB, Lebrilla CB, et al. (2011) Human milk glycobiome and its impact on the infant gastrointestinal microbiota. Proc Natl Acad Sci U S A 108, Suppl. 1, 4653-4658.

28. Davis JC, Totten SM, Huang JO, et al. (2016) Identification of oligosaccharides in feces of breast-fed infants and their correlation with the gut microbial community. Mol Cell Proteomics 15, 2987-3002.

29. Dotz V, Rudloff S, Meyer C, et al. (2015) Metabolic fate of neutral human milk oligosaccharides in exclusively breast-fed infants. Mol Nutr Food Res 59, 355-364.

30. Gnoth MJ, Kunz C, Kinne-Saffran E, et al. (2000) Human milk oligosaccharides are minimally digested in vitro. J Nutr $\mathbf{1 3 0}$, 3014-3020

31. Gnoth MJ, Rudloff S, Kunz C, et al. (2001) Investigations of the in vitro transport of human milk oligosaccharides by a Caco-2 monolayer using a novel high performance liquid chromatography-mass spectrometry technique. J Biol Chem 276, 34363-34370

32. De Leoz ML, Wu S, Strum JS, et al. (2013) A quantitative and comprehensive method to analyze human milk oligosaccharide structures in the urine and feces of infants. Anal Bioanal Chem 405, 4089-4105.

33. Dotz V, Rudloff S, Blank D, et al. (2013) 13C-labeled oligosaccharides in breastfed infants' urine: individual-, structureand time-dependent differences in the excretion. Glycobiology 24, 185-194.

34. Rudloff S, Pohlentz G, Borsch C, et al. (2012) Urinary excretion of in vivo (1)(3)C-labelled milk oligosaccharides in breastfed infants. Br J Nutr 107, 957-963.

35. Rudloff S, Pohlentz G, Diekmann L, et al. (1996) Urinary excretion of lactose and oligosaccharides in preterm infants fed human milk or infant formula. Acta Paediatr 85, 598-603.

36. Underwood MA, Gaerlan S, De Leoz ML, et al. (2015) Human milk oligosaccharides in premature infants: absorption, excretion, and influence on the intestinal microbiota. Pediatr Res 78, 670-677.

37. Ruhaak LR, Stroble C, Underwood MA, et al. (2014) Detection of milk oligosaccharides in plasma of infants. Anal Bioanal Chem 406, 5775-5784

38. Goehring KC, Kennedy AD, Prieto PA, et al. (2014) Direct evidence for the presence of human milk oligosaccharides in the circulation of breastfed infants. PLOS ONE 9, e101692.

39. Bienenstock J, Buck RH, Linke H, et al. (2013) Fucosylated but not sialylated milk oligosaccharides diminish colon motor contractions. PLOS ONE 8, e76236.

40. Urashima T, Saito T, Nakamura T, et al. (2001) Oligosaccharides of milk and colostrum in non-human mammals. Glycoconj J 18, 357-371.

41. Jantscher-Krenn E, Marx C \& Bode L (2013) Human milk oligosaccharides are differentially metabolised in neonatal rats. Br J Nutr 110, 640-650.

42. Santos-Fandila A, Zafra-Gomez A, Vazquez E, et al. (2013) Ultra high performance liquid chromatography-tandem mass spectrometry method for the determination of soluble milk glycans in rat serum. Talanta 118, 137-146.

43. Pickard JM \& Chervonsky AV (2015) Intestinal fucose as a mediator of host-microbe symbiosis. I Immunol 194, 5588-5593. 